\title{
A Practical Guide to Using Time-and-Motion Methods to Monitor Compliance With Hand Hygiene Guidelines: Experience From Tanzanian Labor Wards
}

\author{
Giorgia Gon, ${ }^{a}$ Said M. Ali, ${ }^{b}$ Robert Aunger, ${ }^{a}$ Oona M. Campbell, ${ }^{a}$ Mícheál de Barra, ${ }^{c}$ Marijn de Bruin, d,e

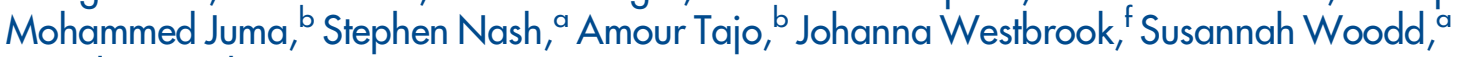 \\ Wendy J. Graham ${ }^{a}$
}

\section{Key Messages}

- Time-and-motion methods are a good way of capturing hand hygiene compliance; for example, they can limit observer bias.

- We describe how we designed the HANDS at Birth tool, the tool format and its elements, its implementation components, the tool's performance, and the implications for data analysis.

- The advantages of using this tool include simpler training, less observer judgment in assessing hand hygiene compliance, and improved ability to monitor multiple behaviors.

\footnotetext{
a London School of Hygiene and Tropical Medicine, London, United Kingdom.

b Public Health Laboratory-lvo de Carneri, Pemba, Zanzibar, Tanzania.

c Brunel University London, Department of Life Sciences, Uxbridge, United Kingdom.

${ }^{d}$ Institute of Applied Health Sciences, University of Aberdeen, Aberdeen, United Kingdom.

e Department of IQ Healthcare, Radboud University Medical Center, Radboud Institute for Health Sciences, Nijmegen, The Netherlands.

${ }^{f}$ Macquarie University, Sydney, Australia.

Correspondence to Giorgia Gon (Giorgia.gon@lshtm.ac.uk).
}

\section{ABSTRACT}

Background: Good-quality evidence on hand hygiene compliance among birth attendants in low-resource labor wards is limited. The World Health Organization Hand Hygiene Observation Form is widely used for directly observing behaviors, but it does not support capturing complex patterns of behavior. We developed the HANDS at Birth tool for direct observational studies of complex patterns of hand rubbing/washing, glove use, recontamination, and their determinants among birth attendants. Understanding these behaviors is particularly critical in wards with variable patient volumes or unpredictable patient complications, such as emergency departments, operating wards, or triage and isolation wards during epidemics. Here we provide detailed information on the design and implementation of the HANDS at Birth tool, with a particular focus on low-resource settings. We developed the HANDS at Birth tool from available guidelines, unstructured observation, and iterative refinement based on consultation with collaborators and pilot results. We designed the tool with WOMBAT soffware, which supports collecting multidimensional time-and-motion data. Our analysis of the tool's performance centered on interobserver agreement and convergent validity and the implications of the data structure for data analysis. The HANDS at Birth tool encompasses various hand actions and context-relevant information. Hand actions include procedures relevant during labor and delivery; hand hygiene or glove actions; and other types of touch. During field implementation, we used the tool for continuous observation of the birth attendant. Interobserver agreement was good (kappa range: $0.7-0.9$ ), and the tool showed convergent validity. Using the HANDS at Birth tool is a feasible way to obtain useful information about compliance with hand hygiene procedures. The tool could be used after simple training and allows for collection of reliable information about the complex pattern of hygiene behaviors. Future studies should explore using this tool to observe behavior in labor wards in other settings and in other types of wards.

\section{BACKGROUND}

nfection prevention is paramount to limiting the spread of epidemics, such as coronavirus disease 2019 (COVID19), severe acute respiratory syndrome, and Ebola, and hand hygiene $(\mathrm{HH})$ is at the forefront of prevention efforts among health care workers. ${ }^{1}$ In addition, health care workers' $\mathrm{HH}$ is essential at the time of birth for preventing 


Time-and-motion
methods can
overcome some of
the challenges
with direct
observation
because they
enable observers
to record all
health care
workers' actions
without having to
decide which ones
represent a new
hand hygiene
opportunity.

health care-associated infections that lead to an enormous burden of illness among mothers and newborns, even in nonepidemic situations. ${ }^{2-4}$ Accurately understanding the specific actions that contribute to the low compliance levels for $\mathrm{HH}$ that occurs in many countries, particularly in lowresource settings, is essential for effective behavior change; yet current tools fail to account for health care workers' workflow and the issue of recontamination and its drivers.

Multiple methods exist to measure HH compliance in health care settings, but observation of behaviors is considered to be the gold standard. ${ }^{5}$ Observation can be done by an observer or by video recording. A recent validation study suggests that both approaches capture similar numbers of $\mathrm{HH}$ opportunities-moments when health care workers ought to practice hand rubbing/washing ${ }^{6}$; however, video recording poses substantial ethical issues, which often makes it difficult to use, particularly in a process such as childbirth when women are vulnerable and undressed. The World Health Organization (WHO) HH Observation Form is an excellent, widely used tool for direct observation. ${ }^{7}$ However, due to its aim and scope, it does not allow capturing more complex patterns of behavior. For example, it does not distinguish whether the failure to comply was because hand rubbing/washing was not attempted or because hands were recontaminated after initial washing. ${ }^{8}$ Avoiding hand/glove recontamination is implicit in the WHO tool's $\mathrm{HH}$ definition because touching a surface carries the risk of germ transmission and creates a new HH opportunity. It also does not aim to capture the use or "misuse" of gloves. ${ }^{9}$ Finally, it requires the observers to judge when a new HH opportunity arises, thereby reducing the consistency of data collection by multiple observers.

Defining when a new HH opportunity arises is particularly difficult in labor and delivery, during which observers must deal with a transition from observing 1 patient (the mother) to 2 (mother and newborn). Furthermore, the amount, type, and location of body fluids can rapidly change during labor and delivery, and in the context of lowresource settings, a single health care worker may attend many mothers simultaneously. With an often unpredictable duration of the different stages of labor, the time between hand rubbing/washing and delivery of the newborn may be lengthy, during which time the observer needs to pay close attention to assess if any actions occur that lead to a new HH opportunity. Time-and-motion methods can overcome some of these challenges. These methods are now at the forefront of health care observation ${ }^{10}$ and are increasingly used, but seldom in low- and middle-income countries. These methods enable observers to record all health care workers' actions without having to decide which ones represent a new HH opportunity. Instead, opportunities are defined during data analysis.

The HANDS study (Hand-hygiene of Attendants for Newborn Deliveries and Survival) was a mixedmethods, cross-sectional research study conducted in the 10 highest volume maternity wards in Zanzibar between November 2015 and April 2017. The aim of the study was to explore compliance with $\mathrm{HH}$ guidelines and identify factors that explain compliance. HH during labor and delivery is a key opportunity to prevent transmitting infections to mothers and newborns ${ }^{3,11}$; however, good-quality evidence on HH compliance from low-resource labor wards is limited. ${ }^{12-16}$ Therefore, we developed the HANDS at Birth tool, based on a time-and-motion design, to observe the complex patterns of birth attendants' $\mathrm{HH}$ and glove use at 3 levels: the opportunity, the individual, and the facility. We designed the tool within WOMBAT software, which is intended to support direct observational studies of health care work. The WOMBAT software package ${ }^{17,18}$ allows collecting multidimensional work tasks, including compliance with specific tasks, and automatically time-stamps data entry. The current investigation was one of the few time-and-motion studies of health care workers conducted with software that automatically records time and carried out in a low-resource setting. ${ }^{19,20}$

Our aim was to provide very practical details regarding the design and implementation of the direct observational tool to measure $\mathrm{HH}$ compliance to inform researchers and practitioners seeking to thoroughly measure the compliance with HH guidelines during labor and delivery, particularly in low-resource settings. In this article, we outline: (1) how we designed the data collection tool, (2) the tool format and its elements, (3) its implementation components, (4) the tool's performance, and (5) the implications for data analysis.

\section{METHODS FOR TOOL DEVELOPMENT}

We developed the HANDS at Birth data collection tool between March and October 2016 using an existing systematic process for tool development. ${ }^{13}$ This process included use of available guidelines, unstructured observation, and iterative refinement based on consultation with collaborators and pilot results.

\section{Guidelines' Review and Semistructured Observation}

We consulted WHO publications, including Hand Hygiene Technical Reference Manual, ${ }^{7}$ Hand Hygiene 
in Outpatient and Home-Based Care and Long-term Care $_{\text {Facilities, }}{ }^{21}$ and Pregnancy, Childbirth, Postpartum and Newborn Care: A Guide for Essential Practice. $^{22}$ We also conducted 11 semi-structured observation sessions in 4 labor wards in Zanzibar during which either a delivery or a vaginal examination occurred. All birth attendants' actions were recorded, together with the time when they happened and their location. Using this information, we created a list of procedures (what we also call "key attendant-patient interactions") relevant to labor and delivery that also included other hand actions that can occur before and after each of these procedures.

\section{Iterative Collaborator Consultation}

The project was a partnership of the London School of Hygiene and Tropical Medicine, the University of Aberdeen, and the Public Health Laboratory of Pemba; we sought feedback on the tool from all project members. Additionally, a 3-hour in-depth consultation was conducted with 2 clinically trained members of the team (1 general practitioner and 1 midwife) who provided additional feedback.

\section{Pilot Activities and Training}

We conducted 3 pilot activities in a labor ward on Pemba Island, Zanzibar, Tanzania. Two data collectors conducted the first pilot in June 2016 using an early version of the HANDS at Birth tool. One data collector conducted the second pilot in August 2016 using the tool incorporated into WOMBAT v2 software on a tablet. Finally, 1 data collector conducted the third pilot in September 2016 using the tool with WOMBAT. Feedback was collected and incorporated to improve the tool at each stage.

Observers were trained to use the tool over 3 days using role-plays and presentations. Each observer also practiced using the tool in the labor ward for 3 hours under trainer supervision (GG). The trainer also conducted 2 hours of observation with each observer and provided them with relevant feedback. During training, minor refinements were made to the tool.

We used the STROBE checklist for crosssectional studies to design and describe this tool here and in other relevant manuscripts including the study results. ${ }^{23}$

The project was approved by the Zanzibar Medical Research and Ethics Committee, the London School of Hygiene and Tropical Medicine Research Ethics Committee, and the Research
Ethics Committee at the University of Aberdeen. Details of procedures to consent are described below.

\section{TOOL FORMAT AND ELEMENTS}

Following Lopetegui et al.'s classification, ${ }^{10}$ our time-and-motion study used continuous observation, in which an external observer focuses on 1 subject, in our case, the birth attendant. When a birth attendant performed an action, the observer recorded the action. We chose to use continuous observation because the timing of procedures, particularly delivery itself, was typically unpredictable, and using alternative methods, such as short observation sessions at fixed or random intervals, could have missed many $\mathrm{HH}$ opportunities. Hence, observers were asked to remain in the labor room for the entirety of their allocated shift (about 7 hours for morning/afternoon shifts and 10 hours for night shifts) and to start recording observations whenever a patient-attendant interaction began.

The tool, available in Supplement 1, includes a list of hand actions and context-relevant information (Figure). The hand actions listed were exhaustive (meaning that the list did not leave any possible actions out) and mutually exclusive (meaning that no 2 actions could occur simultaneously). We did not design a tool that aimed to capture multitasking or interruptions because we did not want to add to the burden on the observers.

Hand actions were either procedures relevant during labor and delivery (e.g., vaginal examination) (Table 1), HH or glove actions, or other types of touches (e.g., touching a pen or equipment). Observers recorded when an attendant left the room where observation was occurring (when observation was suspended) and when the attendant re-entered.

The tool also captured information on the context, such as availability of key infrastructure/ staffing (e.g., water or the presence of the nurse in-charge) and which woman was being attended (first, second, third, etc. since the beginning of the observation session). This process allowed us to assess whether birth attendants performed $\mathrm{HH}$ between patients. Observers entered this contextrelated information at the beginning of the observation session and updated it only if the situation changed.

Many of the recorded actions required further details to be entered. For example, when a delivery was observed, the observer also recorded

\author{
Our time-and- \\ motion study used \\ continuous \\ observation, in \\ which an external \\ observer focuses \\ on one subject, \\ such as a birth \\ attendant.
}


FIGURE. Screen Showing HANDS at Birth Tool to Collect Multidimensional Time-and-Motion Data on Hand Actions and Context-Relevant Information on Hand Hygiene. (Left) Screen That Appears When User Logs In to Tool. (Right) Screen That Appears When User Scrolls Down.

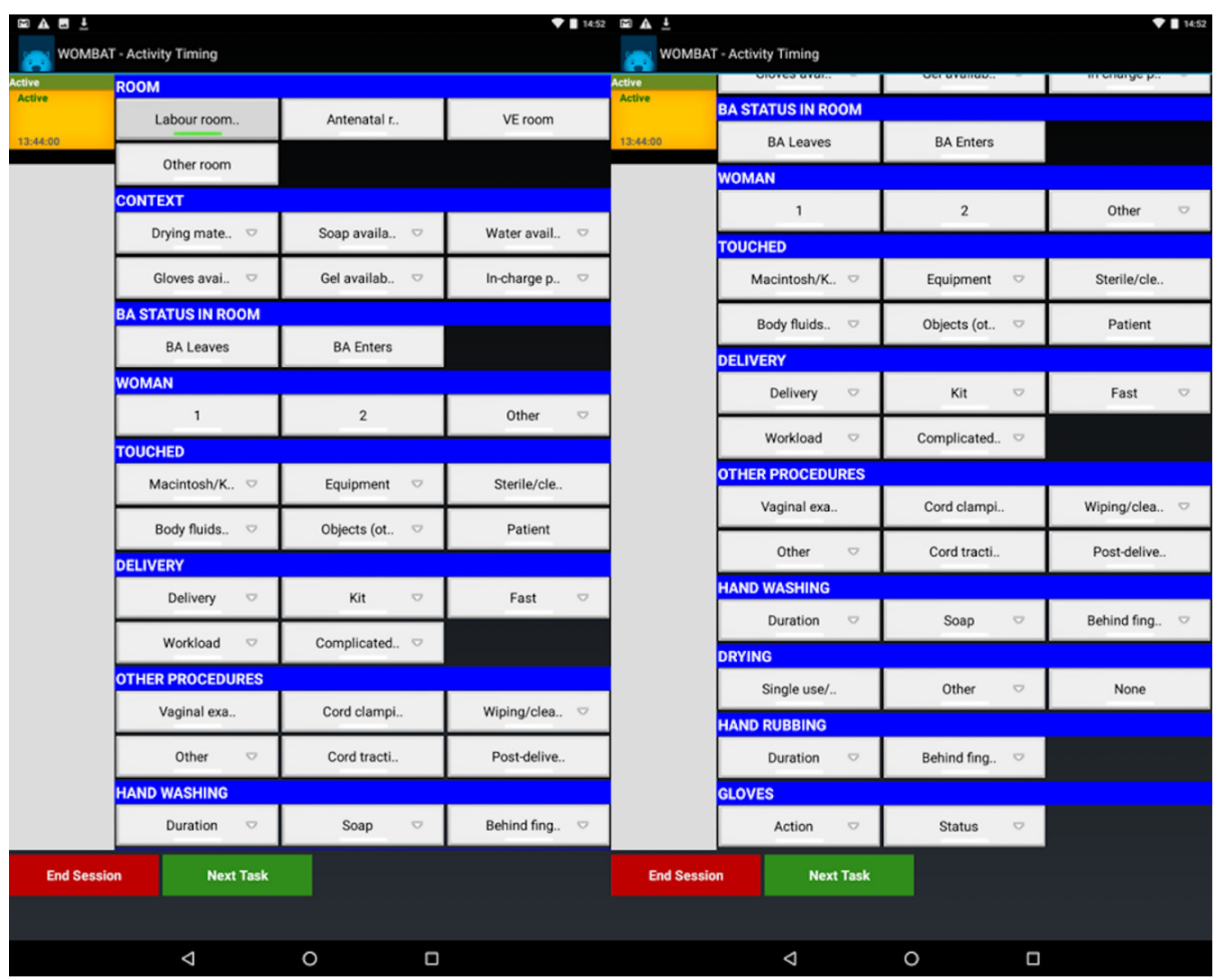

Abbreviations: BA, birth attendant; VE, vaginal examination.

whether the delivery occurred rapidly (within 5 minutes of the woman walking into the labor room), whether there were complications, whether the observer birth attendant had an assistant, and whether a premade delivery kit was used. The observers collected contextual information and details of certain actions because we intended to use these data as potential determinants of $\mathrm{HH}$ in the analysis. The determinants collected and associated with $\mathrm{HH}$ are described in detail in Gon et al. ${ }^{24}$

\section{TOOL IMPLEMENTATION}

This section characterizes how we used the tool to collect data and provides considerations for using it in future studies. We used the guidance provided by Zheng et al. ${ }^{25}$ for reporting time-and-motion studies, and include their full STAMP checklist information in Supplement 2.

\section{Sample Size Calculations}

The data collection timeframe was based on the expected number of deliveries in the targeted facilities. We estimated the latter, using the formula for estimating a proportion from a cross-sectional survey with $\alpha=0.05$ and $80 \%$ power. We used a design effect of 2 based on a survey by Rowe et al. ${ }^{26}$ To estimate a hand rubbing/washing compliance of $10 \%$ with an absolute precision of $\pm 3 \%$, we needed $768 \mathrm{HH}$ opportunities. We estimated the length of observation needed to collect this number, and in practice these data were collected during 336 observation sessions ranging from 13 minutes to 6 hours 45 minutes, with a median time of 1 hour and 41 minutes. ${ }^{8}$ As described in Gon et al, ${ }^{8}$ we collected information on $781 \mathrm{HH}$ opportunities before aseptic procedures (before aseptic procedures is 1 of the 5 types of $\mathrm{HH}$ opportunity prescribed by $\mathrm{WHO}^{7}$ ). 
TABLE 1. Relevant Hand Actions During Labor and Delivery Included in the HANDS at Birth Tool for Observation of Birth Attendants

\begin{tabular}{l}
\hline Measuring vital signs \\
\hline Wiping the vagina \\
\hline Vaginal examination \\
\hline Artificial rupture of membranes \\
\hline Episiotomy \\
\hline Catching the baby (delivery) \\
\hline Cord cutting and clamping \\
\hline Cord traction \\
\hline Postdelivery examination of the vagina \\
\hline Wiping the baby clean after birth \\
\hline Supporting breastfeeding \\
\hline Manual removal of placenta \\
\hline Suturing \\
\hline Suctioning baby's nose/mouth \\
\hline Using bag and mask on the baby \\
\hline Catheter insertion or removal \\
\hline Insertion or removal of IV lines \\
\hline Adjusting IV fluids or changing IV bag \\
\hline Abrigion intrans.
\end{tabular}

Abbreviation: IV, intravenous.

\section{Planning and Logistics of Data Collection}

To obtain representative data on deliveries across all shifts (morning, afternoon, and night); 3 observers, 1 per shift, conducted observations that covered 24 hour a day. They observed for a total of 130 hours in the morning, 153 hours in the afternoon, and 205 hours in the night. Each observer had their own tablet for data collection. Each facility was visited for a mode of 6 consecutive days (range: 5-14 days) between September 17 and December 31, 2016. The order in which we visited the facilities was based on logistics. We arranged for additional days of observation in 1 facility with a high volume of staff to allow all staff to be observed and in 3 facilities with low volume of deliveries to capture a sufficient number of procedures.

We consulted the ward rosters to allocate individual attendants to the observers. Each attendant had a unique identifier that the observer had to record in WOMBAT when observing them. Observers were allocated to shifts based on the following principles: (1) the same observer should observe the same attendant so the attendant becomes accustomed to the same person being on the ward; (2) the initial attendant-observer pairs at each facility were assigned at random (unless specific concerns were raised; e.g., some flexibility on choice of types of shifts was allowed to accommodate observers' needs); and (3) observation days should ideally be planned during changes in shift pattern to allow observation of the same attendants working on different types of shifts. The need to observe the same attendant across different types of shifts using the same observer increased the fieldwork duration and therefore had to be counterbalanced by the need to remain within our budget.

\section{The Observers}

Observers were all trained nurse-midwives working in managerial roles. Two of them worked in the study facilities but not in the labor wards. The third observer worked in district-level management. Their previous knowledge and understanding of the labor process were vital to ensuring quality during data collection and ultimately the project's success.

\section{Study Participants}

All birth attendants present during the observation period who were involved in the childbirth procedures outlined in Table 1 were eligible for observation. We observed a total of 104 birth attendants across the 10 facilities and between 4 and 15 birth attendants in each facility. Each attendant was observed for 1-9 observation sessions. ${ }^{8}$ In each observation session, only 1 attendant was observed, but that attendant could be caring for multiple women and carrying out many procedures. Attendants in our study were all women, 90\% were professionally trained, and $10 \%$ were health orderlies/nonprofessionals.

The attendants' responsibilities were usually allocated during the shift itself. We encouraged observers to listen at staff meetings to learn which attendant was most likely to perform the childbirth procedures outlined in Table 1 to decide whom to observe. Observers were instructed to observe each allocated birth attendant roughly equally in each facility.

\section{How to Observe}

We trained the observers to enter only 1 action at a time to facilitate the data input process. We were specifically interested in the attendants' actions, the sequence of these actions, and the length of time between them, rather than the duration of

\section{Observers were all trained nurse- midwives, whose previous knowledge and understanding of the labor process were vital to the success of the project.}


each action per se. An action was selected and entered immediately. We do not have details on when the action ended, but since the actions were mutually exclusive, it was clear when one action replaced another.

\section{When to Observe}

As described above, a relevant patient-attendant interaction (Table 1) triggered the start of data entry; observers were expected to be continuously present in the ward due to the unpredictable nature of birth. Observers were encouraged to take breaks when no women were in labor or when women were in very early stages of labor and to remain where they could see if an emergency admission occurred to avoid missing delivery events. We also encouraged breaks if the observer's concentration level was low.

We instructed observers to end a session when a major procedure ended and no further patient activities were in sight, when the observer wanted to take a break, when there was the opportunity to start observing another birth attendant, or when the birth attendants left the room to perform duties elsewhere.

\section{Where to Observe}

Observers would usually sit in the labor room. If no deliveries were happening, we asked observers to observe vaginal examinations in other rooms, such as the antenatal ward or examination room.

\section{Consent and Study Aim Concealment}

Written consent was gathered from women in the antenatal ward before observation; alternatively, women were asked for verbal consent once in the labor ward, and follow-up for written consent occurred in the postnatal ward before discharge or before delivery in the antenatal ward. ${ }^{8}$ Women were told that no demographic information was collected on them and recorded observations were exclusively regarding birth attendants' behavior. Permission to observe the attendants was obtained by the Ministry of Health and verbal consent was obtained by the observers when they first visited the facility. ${ }^{8}$

Attendants were told the observation was about the quality of care at birth, not on HH specifically, to conceal the study's focus and reduce the Hawthorne effect. In all but the facility in Zanzibar where piloting took place, the focus of the study (HH practices) was likely to have been well concealed from the birth attendants being observed. The pilot facility in Zanzibar had the highest compliance with hand rubbing/washing before aseptic procedures. Compliance was 10\% higher than the second-best facility and 7 times higher than the worst one. For ethical reasons, observers were trained to notify health workers and the field manager if they observed a potentially harmful condition or practice.

\section{Quality of Data Collection}

To ensure quality of data collection, we held regular meetings with collectors by telephone and onsite, communicated via a WhatsApp group, held Skype calls at the end of observations in each facility, and monitored the data uploaded monthly. These communication channels enabled rapid feedback, answers to questions, and maintenance of morale during long periods of observation. Drivers ensured observers arrived at sites on time. Finally, we are confident that the data were unlikely to have been manufactured because manufacturing time-stamped data would require as much time as conducting and recording actual observations.

\section{Software and Information Technology Costs}

The cost of the software and hardware also needs to be considered especially for deployment in low- and middle-income countries. WOMBAT 3.0 is available from the Apple Store (https://apps.apple.com/us/ app/wombat-3-0/id1445107457). Data hosting is available at a cost of US\$2,500 for a 2-year period, which allows the use of the software for multiple projects and data collectors. Free packages such as Open Data Kit could be used, but Open Data Kit is less user friendly for time-and-motion studies. In addition, we bought 3 tablets for approximately US\$500.

\section{- TOOL PERFORMANCE}

\section{Interobserver (Interrater) Agreement}

To report on interobserver agreement procedures and findings, we followed the recommendations by Lopetegui et al. ${ }^{27}$ for time-and-motion studies and consulted the WOMBAT guidelines. ${ }^{18,28}$ While piloting the tool, the trainer conducted 2 hours of simultaneous observation between the trainer (GG) and each of the observers. We then verified the extent of agreement between GG and each of the 3 observers on the basis of 28, 29, and 36 opportunities for hand washing/rubbing, glove wearing, and touch events, respectively. The observations were based on a total of 11 vaginal 
examinations and 5 deliveries. The exercise was also used to provide feedback to the observers.

During the first month of data collection, we also assessed interobserver agreement, whereby a pair of observers was allocated to 2 of the same shifts in the busiest facility and asked to observe the same attendants. Observers were asked to perform this independently, avoiding communication or looking at each other's tablet, but we could not ensure they were blinded, which meant that they probably knew we were going to check the data and hence some form of communication might still have occurred. Two pairs carried out this exercise for 1 morning and 1 afternoon shift each, the other pair for 2 night shifts. Two pairs observed 3 birth attendants, and the third pair observed 4.

We calculated kappa statistics based on either 49 or 50 hand rubbing/washing, hand recontamination, or glove behaviors per pair of observers. Observations were based on a total of 9 vaginal examinations and 11 deliveries. Through visual inspection of the data, we ensured that the behaviors compared were the same between observers by checking the reported time and sequence of actions. The kappa statistic calculated for pairs of observers was good for 2 of the 3 pairs at 0.93 and 0.90 , but it was below the optimal level of 0.85 for 1 of the pairs at $0.73 .^{18}$ In addition, we are also confident that discrepancies between observers was minimal because our results showed that hand rubbing/washing compliance before aseptic procedures did not vary substantially by observer, as described in Gon et al. ${ }^{8}$

\section{Convergent Validity}

We assessed the degree to which 2 measures of constructs that theoretically should be related were in fact related (convergent validity) by showing whether hand rubbing/washing before aseptic procedures compliance varied in the expected direction by contextual characteristics. Using the methods described in Gon et al, ${ }^{24}$ we descriptively showed that higher compliance was present when the necessary equipment (water and soap or gel) was available, when fewer women were attended in the same observation session (i.e., a lower workload was expected to be associated with better $\mathrm{HH}$ ), and when attendants had received $\mathrm{HH}$ refresher training in the previous year (Table 2).

\section{IMPLICATIONS FOR DATA ANALYSIS} AND INTERPRETATION

In Supplement 3, we describe data cleaning, analysis, and interpretation issues that needed to be considered, noting in particular, that some data

TABLE 2. Hand Rubbing/Washing Compliance Before Aseptic Procedures Among Birth Attendants in Health Facilities in Zanzibar, Tanzania

\begin{tabular}{|c|c|c|}
\hline & $\begin{array}{c}\text { Observed } \\
\text { Opportunities/Indications } \\
\text { for Hand Hygiene, } \\
\text { per Guidelines }{ }^{a} \\
\text { n (\%), N=779 }\end{array}$ & $\begin{array}{c}\text { Hand Hygiene Compliance } \\
\text { (Hands Rubbed/Washed) } \\
\text { When Indicated } \\
\text { n (\%), N=190 }\end{array}$ \\
\hline \multicolumn{3}{|c|}{ Necessary hand hygiene equipment (water and soap or gel) } \\
\hline No & $48(6.2)$ & $5(10.4)$ \\
\hline Yes & $704(90.4)$ & $177(25.1)$ \\
\hline Missing & $13(1.7)$ & $3(23.1)$ \\
\hline Inconsistent information & $14(1.8)$ & $5(35.7)$ \\
\hline \multicolumn{3}{|c|}{ Maximum number of women attended in an observation session } \\
\hline 1 & $541(69.5)$ & $146(27.0)$ \\
\hline 2 & $196(25.2)$ & $39(19.9)$ \\
\hline 3 & $36(4.6)$ & $4(11.1)$ \\
\hline Missing & $6(0.8)$ & $1(16.7)$ \\
\hline \multicolumn{3}{|c|}{ Hand hygiene refresher training in the past 12 months } \\
\hline No & $347(44.5)$ & $74(21.3)$ \\
\hline Yes & $432(55.5)$ & $116(26.9)$ \\
\hline
\end{tabular}

a Number of times when hand hygiene was meant to be performed per guidelines. 


\section{A strength of WOMBAT is that when each action is recorded, the time of that action is automatically logged.}

items relied on observer subjectivity (e.g., duration of hand washing) and some variables (e.g., variables describing the context) required more stringent training than others.

\section{Data Structure}

A strength of WOMBAT is that when each action is recorded, the time of that action is automatically logged. Our final dataset was a list of 7,893 timeordered entries. These data were coded to derive $\mathrm{HH}$ opportunities and to calculate compliance. First, each HH opportunity needed to be identified within each observation session, which is further explained below. Second, for HH opportunities before aseptic procedures or touching the patient, the sequence of actions preceding the opportunity needed to be examined for hand rubbing/washing actions, glove use and actions that may lead to a new HH opportunity. Whereas, for HH opportunities after exposure to body fluids or touching the patient or the patient's surrounding, the actions following the opportunity needed to be examined. We used STATA to analyze these data.

\section{Time Stamps}

We used WOMBAT's time stamp information in 2 ways. First, we checked the plausibility of certain actions being linked; for example, a hand rubbing/ washing action could not be linked to a procedure conducted 10 hours before or after it. Second, we calculated the length of time between hand rubbing/washing and the $\mathrm{HH}$ opportunity to determine whether time would predict the likelihood of hand recontamination occurring.

\section{A Priori Definitions Required}

To estimate HH compliance, we operationalized definitions for the systematic flow of patient contacts allowed within a given HH opportunity and the patient zone. By a systematic flow, which we called a "delivery flow," we referred to the procedures or actions of interest that defined the start of a new HH opportunity, as well as the sequence of these procedures, which occurred without a break and were considered as 1 opportunity for $\mathrm{HH}^{21}{ }^{21}$ For example, in a given delivery flow, a vaginal examination could be followed by the delivery of the baby, but not by touching a patient's shoulder. During a delivery flow, a birth attendant could undertake hand actions within the patient zone without the need for a new $\mathrm{HH}$ opportunity to arise.

In this study, we defined patient zone as encompassing a woman's perineal area and thighs, any clean or sterile equipment being used, and the newborn as it was caught and wiped. A break in the delivery flow, indicating a new HH opportunity, arose if an activity occurred that was outside the patient zone, such as inserting an intravenous line, touching the patient beyond the zone, or leaving the room. ${ }^{8}$

Details on the definitions used in our study are reported in Gon et al. ${ }^{8}$ Potentially, a separate software could be programmed to automatically analyze this type of data in the future, allowing for definitions to be applied from the outset.

\section{Context-Specific Adaptations}

To classify which surfaces we should include in the patient zone, we used previous formative research $^{29}$ on the microbiological load of the labor surfaces in Zanzibar, as well as unstructured observation of labor wards conducted within the HANDS project. For example, we excluded the delivery bed and trolley from the patient zone because previous work found that these surfaces were often contaminated with potential pathogens. ${ }^{8}$ Other important information to consider include the details of the cloth or plastic sheet used under the woman's body during birth, the cleaning routines of the wards, the type of water available, the delivery equipment preparation, and the local HH guidelines against which to measure hand washing/rubbing duration and technique. It is not clear that all projects will have the capacity to gather this level of contextual information; however, capturing the real workflows in this context was our aim.

Ideally, all definitions should be clear at the start of a project, but during data collection, the project may accrue context-specific information on the surfaces or the attendants' workflows, which should be used to update the definitions. To illustrate this, we present the number of $\mathrm{HH}$ opportunities and hand rubbing/washing compliance results for 4 different patient zone definitions (Supplement 4).

\section{DISCUSSION}

We developed the HANDS at Birth tool to capture the complex $\mathrm{HH}$ and glove behaviors of birth attendants, based on state-of-the-art methods: a time-and-motion study using a computerized system (WOMBAT). This approach has been rarely used to measure $\mathrm{HH}$ or to conduct research in low-resource settings. ${ }^{10,19,20,30,31}$ Our time-andmotion study enabled us to accomplish the following, which would have not been possible with the 
WHO HH Observation Form: (1) to look at whether birth attendants comply with the complete sequence of behaviors prescribed by the WHO guidelines, $^{32}$ (2) to look at each behavior individually, and (3) to look at different behavior sequences. 8 Additionally, our method reduced the risk of observer bias because data collection was coded as a series of individual actions rather than relying on observer judgment that a new $\mathrm{HH}$ opportunity had occurred; hence, opportunities were identified retrospectively in a standardized way. ${ }^{33}$ Indeed, hand rubbing/washing compliance was similar between observers in our study, as reported in Gon et al. ${ }^{8}$ Beyond $\mathrm{HH}$, the HANDS at Birth tool allowed investigation of other behavior sequences and workflows.

We are aware of 1 other study that used timeand-motion methods to report $\mathrm{HH}$ of health care workers in the context of an intensive care unit in the United States. ${ }^{31}$ That study's aims differed from ours including determining the number of contacts between patients and health care workers, as well as how long they take, and estimating $\mathrm{HH}$ compliance specifically before entering a room and after exiting a room. That study did not detail information on the tool format or content. In comparison, the HANDS at Birth tool allows for a more exhaustive list of actions to be recorded, including those beyond patient-attendant interactions; it also allows looking at all $\mathrm{HH}$ opportunities, not just those related to exiting or entering the room.

This tool has the potential to be adapted to examine $\mathrm{HH}$ in other types of wards. We think this detailed examination of $\mathrm{HH}$, including recontamination, is particularly important in wards facing unpredictable volumes of patients or unpredictable patient complications. Examples include emergency departments, operating wards, or isolation wards during epidemics, such as the current isolation wards for COVID-19 patients. In particular during the COVID-19 pandemic, this tool could lend itself to examining the key relationship between hands and surfaces and the fundamental issue of pathogen cross-contamination between them. ${ }^{34,35}$

\section{Limitations}

Because we were interested in individual determinants of $\mathrm{HH}$ behavior, we observed only 1 birth attendant at any 1 time; whereas, the WHO $\mathrm{HH}$ Observation Form audit tool is designed to observe multiple health care workers simultaneously, which allows collection of more $\mathrm{HH}$ opportunities in the same observation session. Importantly, the HANDS at Birth tool is not intended to substitute for the WHO $\mathrm{HH}$ Observation Form; the 2 tools serve very different purposes, with the former being aimed at research and the latter at infection prevention practitioners. Another limitation of our tool, and how we used it, is that it requires data cleaning and data management. For example, even though misclassification was minimal, some actions were recorded by mistake at the same time. In addition, a couple of variables relied on observer subjectivity-for example whether a delivery happened very fast after the woman's admission in the labor room. The structure of the data implies that data management is needed to create $\mathrm{HH}$ opportunities and $\mathrm{HH}$ compliance results.

\section{CONCLUSION}

In conclusion, we report the process of developing a research tool to capture the complexity of $\mathrm{HH}$ and glove behavior during labor and delivery, including the tool elements, field implementation, tool performance, and implications for analysis. We used a computerized system that was feasible to use in low-resource facilities. Advantages of this tool include simpler training, less observer bias in assessing $\mathrm{HH}$ compliance (compared with the WHO HH Observation Form), and the ability to monitor multiple behaviors. The data it produced also showed good reliability and convergent validity. Future studies should explore the use of this research tool in labor wards in other contexts, as well as in other types of wards.

Acknowledgments: We thank the Ministry of Health of Zanzibar for their participation and engagement in the study. A special thanks to Rukaiya M Said, Mwanafatima Ali Mohammed, Bijuma Mkubwa Abdallah, and Asya Hati Vuai who collected all the data. We also thank Marina Daniele for participating in the consultation exercise aimed at refining the definition of hand hygiene opportunity.

Funding: The project was funded by the United Kingdom Research \& Innovation Medical Research Council (MRC) Public Health Intervention Development scheme (award number MR/N015975/1). This award is jointly funded by the MRC and the United Kingdom Department for International Development (DFID) under the MRC/DFID Concordat agreement and is also part of the European \& Developing Countries Clinical Trials Partnership program supported by the European Union. The Soapbox Collaborative also contributed by funding staff involved in this project.

Competing interests: None declared.

Data Availability: The dataset generated during the current study is available at: https://doi.org/10.17037/DATA.00000778.

\section{REFERENCES}

1. Bedford J, Enria D, Giesecke J, et al.; WHO Strategic and Technical Advisory Group for Infectious Hazards. COVID-19: towards controlling of a pandemic. Lancet. 2020;395(10229):1015-1018. CrossRef. Medline

2. Zaidi AKM, Huskins WC, Thaver D, Bhutta ZA, Abbas Z, Goldmann DA. Hospital-acquired neonatal infections in developing countries. Lancet. 2005;365(9465):1175-1 188. CrossRef. Medline

\section{Our method reduced the risk of observer bias because data collection was coded as individual actions rather than relying on observer judgment that a new HH opportunity had occurred.}


3. Gould IM. Alexander Gordon, puerperal sepsis, and modern theories of infection control-Semmelweis in perspective. Lancet Infect Dis. 2010;10(4):275-278. CrossRef. Medline

4. Allegranzi B, Nejad SB, Combescure C, et al. Burden of endemic health-care-associated infection in developing countries: systematic review and meta-analysis. Lancet. $2011 ; 377(9761): 228-241$. CrossRef. Medline

5. Arai A, Tanabe M, Nakamura A, et al. Utility of electronic hand hy giene counting devices for measuring physicians' hand hygiene adherence applied to outpatient settings. Am J Infect Control. 2016; 44(12):1481-1485. CrossRef. Medline

6. Diller T, Kelly JW, Blackhurst D, Steed C, Boeker S, McElveen DC. Estimation of hand hygiene opportunities on an adult medical ward using 24-hour camera surveillance: validation of the HOW2 Benchmark Study. Am J Infect Control. 2014;42(6):602-607. CrossRef. Medline

7. World Health Organization (WHO). Hand Hygiene Technical Reference Manual. WHO; 2009. Accessed September 8, 2020. http://apps.who.int/iris/bitstream/handle/10665/44196/ 9789241598606_eng.pdf

8. Gon G, de Bruin M, de Barra $M$, et al. Hands washing, glove use, and avoiding recontamination before aseptic procedures at birth: a multicenter time-and-motion study conducted in Zanzibar. Am J Infect Control. 2019;47(2):149-156. CrossRef. Medline

9. Wilson J, Prieto J, Singleton J, O'Connor V, Lynam S, Loveday H. The misuse and overuse of non-sterile gloves: application of an audit tool to define the problem. J Infect Prev. 2015;16(1):24-31. CrossRef. Medline

10. Lopetegui M, Yen PY, Lai A, Jeffries J, Embi P, Payne P. Time motion studies in healthcare: what are we talking about? J Biomed Inform. 2014;49:292-299. CrossRef. Medline

11. Blencowe H, Cousens S, Mullany LC, et al. Clean birth and postnata care practices to reduce neonatal deaths from sepsis and tetanus: a systematic review and Delphi estimation of mortality effect. BMC Public Health. 2011;11 (Suppl 3):S11. CrossRef. Medline

12. Shehu NY, Onyedibe K, Okolo M, et al. Assessment of hand hygiene compliance among health care workers in a Nigerian Tertiary Hospital. Antimicrob Resist Infect Contr. 2017;6(Suppl 3):P101 Meeting abstracts from the International Conference on Prevention \& Infection Control (ICPIC 20170). CrossRef

13. Spector JM, Agrawal P, Kodkany B, et al. Improving quality of care for maternal and newborn health: prospective pilot study of the WHO safe childbirth checklist program. PLoS One. 2012;7(5): e35151. CrossRef. Medline

14. Yawson AE, Hesse AAJ. Hand hygiene practices and resources in a teaching hospital in Ghana. J Infect Dev Ctries. 2013;7(04):338347. CrossRef. Medline

15. Danda G, Dube K, Dube P, Mudokwenyu-Rawdon C, Bedwell C. An observational study of midwives' practices to prevent peripartum sepsis in Zimbabwe. Afr J Midwifery Womens Health. 2015; 9(1):17-21. CrossRef

16. Simbar M, Ghafari F, Tork Zahrani S, Alavi Majd H. Assessment of quality of midwifery care in labor and delivery wards of selected Kordestan Medical Science University hospitals. Int J Health Care Qual Assur. 2009;22(3):266-277. CrossRef. Medline

17. Westbrook Jl, Ampt A. Design, application and testing of the Work Observation Method by Activity Timing (WOMBAT) to measure clinicians' patterns of work and communication. Int J Med Inform. 2009;78(Suppl 1):S25-S33. CrossRef. Medline

18. Westbrook J. Work Observation Method By Activity Timing: A Guide to the Installation and Use of WOMBAT V2. Centre for Health Systems and Safety Research, Macquarie University; 2016.

19. Singh S, Upadhyaya S, Deshmukh $P$, et al. Time motion study using mixed methods to assess service delivery by frontline health workers from South India: methods. Hum Resour Health. 2018;16(1):17. CrossRef. Medline

20. Manzi F, Schellenberg JA, Hutton G, et al. Human resources for health care delivery in Tanzania: a multifaceted problem. Hum Resour Health. 2012;10(1):3. CrossRef. Medline

21. World Health Organization (WHO). Hand Hygiene in Outpatient and Home-Based Care and Long-Term Care Facilities. WHO; 2012. Accessed September 8, 2020. http://apps.who.int/iris/bitstream/ handle/10665/78060/9789241503372_eng.pdf

22. World Health Organization (WHO). Pregnancy, Childbirth, Postpartum and Newborn Care: A Guide for Essential Practice. 3rd ed. WHO; 2015. Accessed September 8, 2020. http://apps. who. int/iris/bitstream/handle/10665/249580/9789241549356eng.pdf

23. STROBE Initiative Group. STROBE checklist for cohort, case-control, and cross-sectional studies (combined). Published October 2007 Accessed September 8, 2020. https://www.strobe-statement.org/ index.php

24. Gon G, Virgo S, de Barra M, et al. Behavioural determinants of hand washing and glove recontamination before aseptic procedures at birth: a time-and-motion study and survey in Zanzibar labour wards. Int J Environ Res Public Health. 2020;17(4):1 438. CrossRef. Medline

25. Zheng K, Guo MH, Hanauer DA. Using the time and motion method to study clinical work processes and workflow: methodological inconsistencies and a call for standardized research. J Am Med Inform Assoc. 2011;18(5):704-710. CrossRef. Medline

26. Rowe AK, Lama M, Onikpo F, Deming MS. Design effects and intraclass correlation coefficients from a health facility cluster survey in Benin. Int J Qual Health Care. 2002;14(6):521-523. CrossRef. Medline

27. Lopetegui MA, Bai S, Yen P-Y, Lai A, Embi P, Payne PRO. Inter-observer reliability assessments in time motion studies: the foundation for meaningful clinical workflow analysis. AMIA Annu Symp Proc. 2013;2013:889-896. Medline

28. Kottner J, Audigé L, Brorson S, et al. Guidelines for Reporting Reliability and Agreement Studies (GRRAS) were proposed. J Clin Epidemiol. 2011;64(1):96-106. CrossRef. Medline

29. Gon G, Ali SM, Towriss C, et al. Unpacking the enabling factors for hand, cord and birth-surface hygiene in Zanzibar maternity units. Health Policy Plan. 2017;32(8):1220-1228. CrossRef. Medline

30. Tipping MD, Forth VE, Magill DB, Englert K, Williams MV. Systematic review of time studies evaluating physicians in the hospital setting. J Hosp Med. 2010;5(6):353-359. CrossRef. Medline

31. Khan BA, Hui KY, Hui SL, et al. Time-motion analysis of health care workers' contact with patients and workers' hand hygiene: open vs closed units. Am J Crit Care. 2011 ;20(3):e75-e79. CrossRef. Medline

32. World Health Organization (WHO). WHO Guidelines on Hand Hygiene in Health Care: First Global Patient Safety Challenge Clean Care Is Safer Care. WHO; 2009. Accessed September 8, 2020. https://apps.who.int/iris/bitstream/handle/10665/44102/ 9789241597906_eng.pdf

33. Muller MP. Measuring hand hygiene when it matters. Lancet Infect Dis. 2016;16(12):1306-1307. CrossRef. Medline

34. Gon G, Dancer S, Dreibelbis R, Graham WJ, Kilpatrick C. Reducing hand recontamination of healthcare workers during COVID-19. Infect Control Hosp Epidemiol. 2020:1-2. CrossRef. Medline

35. Otter J. Considering the role of environmental contamination in the spread of COVID-19. Reflections on Infection Prevention and Control blog. Published March 12, 2020. Accessed September 8, 2020. https://reflectionsipc.com/2020/03/12/considering-the-role-ofenvironmental-contamination-in-the-spread-of-covid-19/ 


\section{Peer Reviewed}

Received: May 13, 2020; Accepted: August 19, 2020; First published online: October 15, 2020

Cite this article as: Gon G, Ali SM, Aunger R, et al. A practical guide to using time-and-motion methods to monitor compliance with hand hygiene guidelines: experience from Tanzanian labor wards. Glob Health Sci Pract. 2020;8(4):827-837. https://doi.org/10.9745/GHSP-D-20-00221

(c) Gon et al. This is an open-access article distributed under the terms of the Creative Commons Attribution 4.0 International License (CC BY 4.0), which permits unrestricted use, distribution, and reproduction in any medium, provided the original author and source are properly cited. To view a copy of the license, visit http://creativecommons.org/licenses/by/4.0/. When linking to this article, please use the following permanent link: https://doi.org/ 10.9745/GHSP-D-20-00221 